\title{
La leucospermie en routine
}

\author{
A. CHOCAT
}

CHU de Caen et Laboratoire Malherbe, Secteur Biologie de la Reproduction, Caen

RÉSUMÉ

Chez l'homme, des leucocytes, le plus souvent des polynucléaires neutrophiles, sont présents dans la plupart des éjaculats. La présence en grand nombre de ces leucocytes, évoque une inflammation du tractus génital, d'origine infectieuse ou non. La quantification en routine de ces cellules n'est pas aisée ; elle s'appuie sur la conjonction des résultats de l'analyse du sperme à l'état frais, et après détection des peroxydases intracellulaires caractérisant les polynucléaires, puis sur frottis coloré.

La présence d'une proportion élevée de leucocytes altérés évoque fortement une infection. Si un grand nombre de leucocytes dans le sperme traduit un état inflammatoire, le rôle de ces granulocytes, lorsqu'ils sont peu nombreux, reste encore sujet à discussion. Une leucospermie doit être interprétée dans le contexte clinique et biologique du patient en s'assurant de la qualité de la communication entre les différents intervenants.

Mots clés : Leucospermie, infertilité masculine, infection, inflammation

\section{INTRODUCTION}

En plus des spermatozoïdes normalement présents, le sperme contient presque toujours des cellules qualifiées de rondes du fait de leur morphologie. Il s'agit soit de cellules germinales immatures soit de leucocytes [7]. Chez l'homme, des leucocytes sont présents dans la plupart des éjaculats ; ils proviennent des voies excrétrices séminales et surtout des glandes annexes, vésicules séminales et prostate.

Les différentes sous-populations leucocytaires peuvent être retrouvées dans des proportions différentes dans le sperme des sujets fertiles ou infertiles.

En général, les polynucléaires neutrophiles représentent environ 50 à $60 \%$ des leucocytes du sperme, les macrophages 20 à $30 \%$ et les lymphocytes seulement 2 à $5 \%$ [9]. Les polynucléaires éosinophiles et basophiles sont très rarement observés.

Selon les normes définies par l'OMS, “ un éjaculat normal ne devrait pas contenir plus de 5 millions/ml de cellules rondes, la concentration en leucocytes ne devant pas excéder 1 mil-

Correspondance : Dr. A. Chocat, Laboratoire Malherbe, Secteur Biologie de la Reproduction, 1 et 5, rue EcuyèreBP 187, 14011 CAEN Cedex

Communication aux 3èmes Journées Nationales Assurance de Qualité en Biologie de la Reproduction, 26 \& 27 Octobre 2000, Nancy. 
lion/ml. " [8]. La prévalence de la leucospermie définie selon ces critères est estimée comprise entre 10 et $20 \%$ chez les hommes consultant pour infertilité [4]

\section{MISE EN ÉVIDENCE}

\section{EXAMEN DIRECT}

Le sperme analysé doit être pur. Avant de suspecter une participation urothéliale, la présence de nombreuses cellules épithéliales doit faire évoquer une souillure (de différentes muqueuses, vagin, bouche, gland..). Un contrôle sur un nouveau prélèvement doit être effectué avec interrogatoire du patient précédant le recueil.

L'observation d'une goutte calibrée de sperme frais lors du spermogramme permet déjà de suspecter la présence de leucocytes. Après avoir bien homogénéisé le sperme, une goutte de $10 \mu \mathrm{l}$ est déposée entre lame et lamelle $22 \mathrm{x}$ $32 \mathrm{~mm}$, et observée en contraste de phase négatif, au grossissement $\times 400$ (Figure 1).

Les polynucléaires neutrophiles sont des cellules de 12 à $14 \mu \mathrm{m}$ de diamètre avec un cytoplasme granuleux très réfringent. Il est possible, en faisant varier la vis micrométrique, d'observer de fines expansions membranaires correspondant à des pseudopodes, le polynucléaire étant doué d'une activité amiboïde.

Le macrophage est une cellule beaucoup plus grosse que le polynucléaire, (souvent plus de $20 \mu \mathrm{m}$ de diamètre), dont le cytoplasme est chargé de granulations nombreuses et variables et qui peut présenter également des pseudopodes.

Le lymphocyte est une cellule difficile à différencier des spermatocytes. Elle a un diamètre compris entre 7 et $12 \mu \mathrm{m}$. Il est quelquefois possible d'observer des pseudopodes souvent regroupés à un pôle lorsque la cellule est activée.

\section{2. ÉTUDES DE FROTTIS}

De la qualité du frottis dépendra la qualité de la lecture. Une goutte de sperme calibrée $(5 \mu \mathrm{l})$ est déposée sur une lame. En approchant une lamelle de 22 × $22 \mathrm{~mm}$ suffisamment près du dépôt, le sperme vient épouser le bord de cette lamelle. L'étalement se fait en tirant la lamelle, emmenant ainsi uniquement la quantité de sperme ayant adhérée. Cette précaution permet un étalement fin, avec un front net en queue de frottis ; les cellules n'ont pas été écrasées par la lamelle ni perdues, poussées en fin de lame. Cet étalement est un reflet fidèle de la cellularité du sperme. La coloration peut se faire en Papanicolaou ; les cytologistes préfèrent la coloration par le May-GrunwaldGiemsa qui permet une observation plus fine de la chromatine des noyaux. Les polynucléaires ont un noyau polylobé, 3 à 5 lobes (d'autant plus de lobes qu'ils sont âgés). Il est important de noter l'état de ces cellules, si elles sont bien conservées ou au contraire altérées avec des noyaux nus, picnotiques, fragmentés et des débris de cytoplasme. Il faut également noter la présence d'agrégats de polynucléaires altérés, très évocateurs. Cet aspect est en faveur d'une pyospermie. (Figure 2). Il est important de ne pas employer ce terme si les polynucléaires, même très nombreux, ne sont pas altérés. La pyospermie est réservée à la présence de pus dans le sperme ; elle s'inscrit souvent dans un tableau clinique évocateur d'une pathologie aiguë (urétrite, prostatite, épididymite ...)

Le macrophage a une morphologie et une affinité tinctoriale très variable (Figures 3,4 et 5 ). Sa chromatine est fine et dispersée. La participation macrophagique est en faveur du caractère évolutif de l'inflammation.

Le noyau du lymphocyte est sphérique ou réniforme à chromatine dense, mottée, gommée. Le cytoplasme est petit, basophile avec quelques granulations azurophiles. Il reste difficile à différencier du spermatocyte.

\section{MÉThOdes DE dÉ'TeCTION PLUS SPÉCIFIQUES}

Plusieurs techniques de quantification des leucocytes dans le sperme ont été proposées. Une technique biochimique mesure, à l'aide d'un test d'immuno-adsorption enzymatique Elisa [10], la concentration d'élastase dans le liquide séminal, reflet indirect de la concentration en polynucléaires neutrophiles.

Une technique immunocytochimique détecte les leucocytes par des anticorps spécifiques 


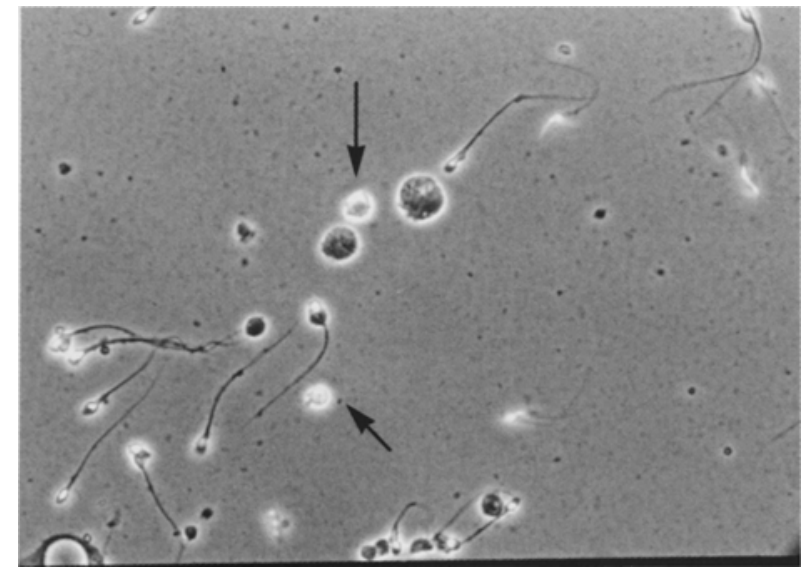

Figure 1 : sperme frais

Les 2 flèches désignent chacune un polynucléaire neutrophile au cytoplasme réfringent, observés en contraste de phase à l'examen direct sur une goutte de sperme frais au grossissement $x$ 400.

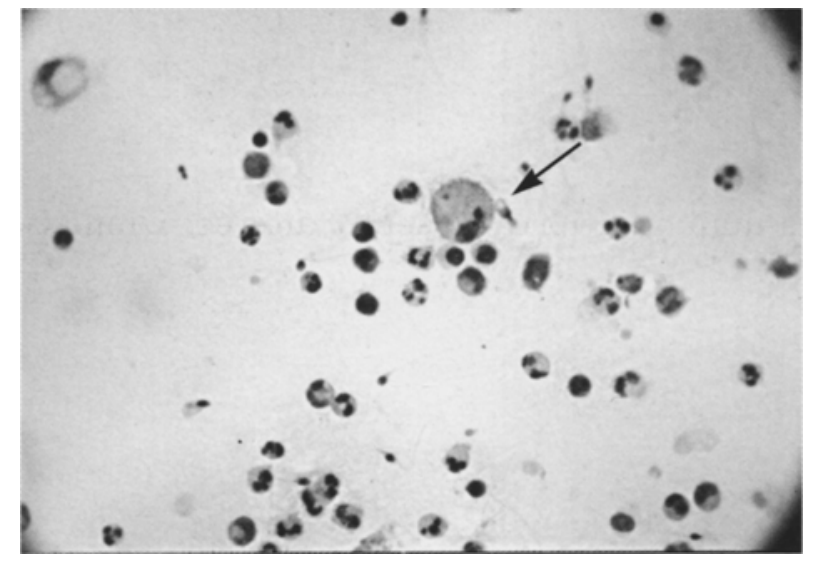

Figure 3 : Frottis colorés en Papanicolaou : macrophage et polynucléaires.

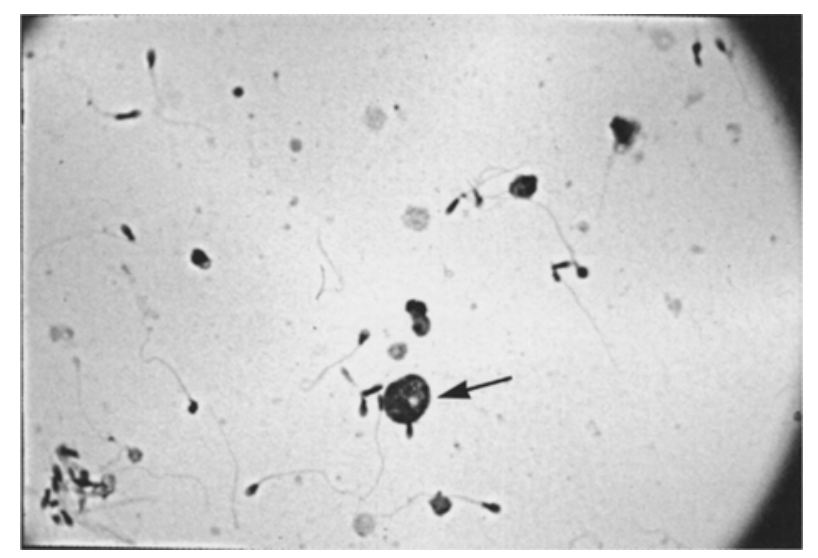

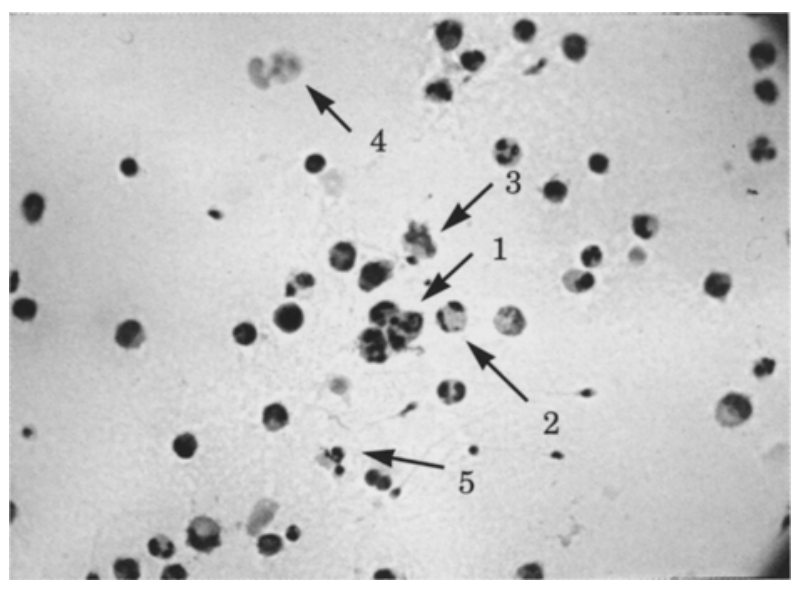

Figure 2 : Pyospermie

Flèche 1 : agrégat de polynucléaires altérés.

Flèche 2 : polynucléaire neutrophile au noyau fragmenté.

Flèche 3 : polynucléaire neutrophile en voie de lyse.

Flèche 4 : restes cytoplasmiques.

Flèche 5 : noyau nu fragmenté.

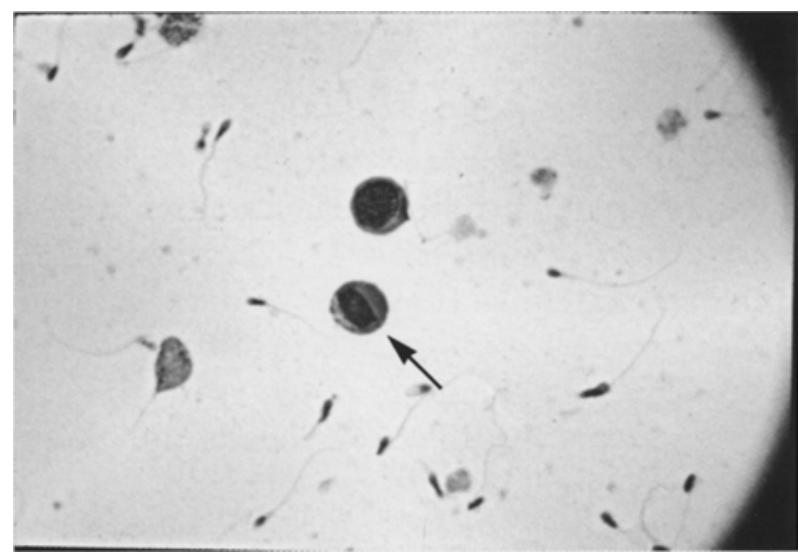

Figure 4 : Frottis coloré en MGG : macrophage.

Figure 5 : Frottis coloré en MGG : autre aspect d'un macrophage. 
monoclonaux CD4 dirigés contre l'antigène leucocytaire commun. Cette technique permet la séparation des cellules sanguines et des cellules germinales immatures [2]. Ces deux techniques sont fiables, mais elles sont lourdes dans leur mise en œuvre et onéreuses ; elles ne peuvent pas être utilisées en routine.

La technique la plus couramment utilisée en routine est une technique cytochimique basée sur la révélation de la peroxydase qui caractérise les polynucléaires ; au contact d'eau oxygénée et de diaminobenzidine, les polynucléaires se révèlent par la présence de granulations brunes [11].

Un kit de réactif est commercialisé sous le nom de Leucoscreen* (JCD)

réactif 1 : contient de la benzidine-cyanosine et du méthanol

réactif 2 : eau oxygénée à $30 \%$

Ces deux réactifs doivent être mélangés extemporanément pour constituer la solution de travail. La réaction a lieu en mélangeant, volume à volume le sperme et la solution de travail dans un tube à hémolyse car la réaction entraîne un dégagement gazeux. Après quelques minutes, on prend une goutte de $10 \mu \mathrm{du}$ mélange au fond du tube, afin ne pas être gêné par les bulles, et on observe cette goutte entre lame et lamelle (lamelle de $22 \times 32 \mathrm{~mm}$ pour un étalement très fin). La lecture se fait sur 100 cellules ; celles qui présentent une coloration jaune à brun sont des cellules à activité péroxydasique positive, les autres présentant une coloration rose (Figure 6). Cette technique

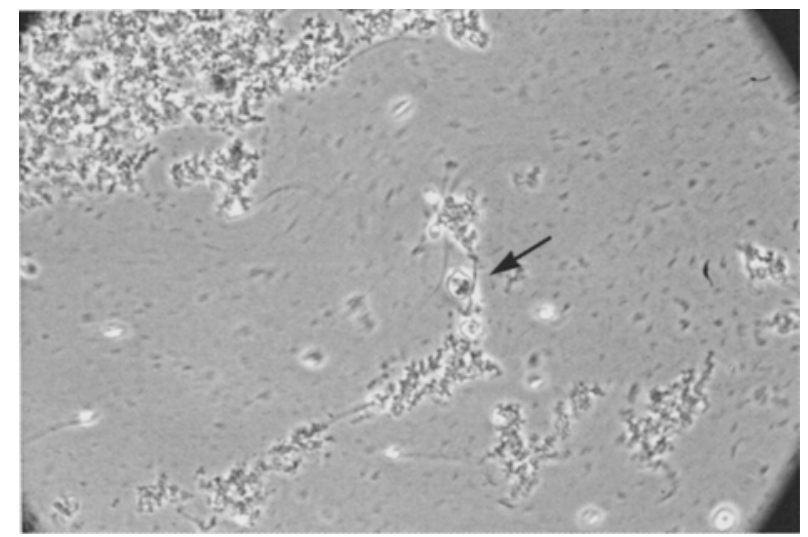

Figure 6 : Coloration des péroxydases intra-polynucléaires. bien que simple et fiable présente quelques inconvénients. En effet, elle ne détecte pas les polynucléaires neutrophiles dégranulés et peu ceux qui sont partiellement dégranulés. Elle ne permet pas non plus de différencier les lymphocytes et les macrophages qui sont dépourvus de granulations péroxydasiques comme les cellules germinales. Il est important de toujours confronter les résultats de cette coloration avec les analyses de l'examen direct et des frottis.

\section{SIGNIFICATION- CONSEQUENCES}

Il semblerait qu'il n'y ait pas de corrélations entre le nombre des leucocytes et celui des cellules germinales immatures [5]. Ceci confirme que la présence, en quantité excessive, de l'une ou l'autre des familles de cellules, n'a pas la même signification physiologique et confirme l'importance de leur identification précise.

Ainsi qu'il est énoncé dans le manuel de laboratoire de l'OMS, " il est difficile de définir un seuil de la concentration en leucocytes au-dessus duquel la fertilité serait altérée. L'impact de ces cellules dépend du niveau du tractus génital où les leucocytes pénètrent dans le sperme, du type de leucocytes présents et leur état d'activation ".

Les polynucléaires sont riches en péroxydase, ce qui a pour effet de faire apparaître de l'oxygène actif qui entraîne des péroxydations de lipides insaturés. Or ces lipides jouent un rôle important dans la composition de la membrane. Il semblerait que, plus il y a de leucocytes producteurs de radicaux oxydatifs (DAO dérivés actifs de l'oxygène, appelés ROS en anglais) dans le sperme, plus l'action néfaste de ces DAO sur les spermatozoïdes est importante [1].

La présence d'une proportion élevée de leucocytes dans le sperme traduit un état inflammatoire (pas forcément d'origine infectieuse). Lorsqu'il y a grand nombre de granulocytes altérés dans le sperme, cela évoque fortement une infection. Le rôle de ces granulocytes, lorsqu'ils sont peu nombreux, reste encore sujet à discussion. Certains leurs attribuent un rôle favorable sur l'élimination de cellules germinales anormales [3]. 


\section{CONDUITE À TENIR}

L'attitude préconisée par l'OMS consiste, lorsque le sperme contient plus d'1 million de leucocytes par $\mathrm{ml}$, à pratiquer des analyses pour tenter de mettre en évidence un agent infectieux.

Le bilan bactériologique comprend une spermoculture avec recherche de chlamydia par PCR et de mycoplasme, et un ECBU avec éventuellement une épreuve de Meares-Stamey, permettant, avec le massage prostatique, d'isoler un germe situé au niveau de la glande prostatique.

Tout en sachant que la mise en évidence de germes à la spermoculture ne s'associe pas toujours à une leucospermie et inversement que l'on peut observer des leucospermies sans infection du sperme.

Une analyse biochimique du plasma séminal peut être réalisée car une infection des glandes annexes est souvent responsable d'une perturbation de la fonction sécrétoire, tout en sachant que l'absence de leucocyte n'exclut pas une infection des glandes annexes.

Enfin, une prise en charge clinique s'impose pour compléter le bilan avec une échographie de l'appareil génital à la recherche d'images évocatrices (calcifications intra-prostatiques, kystes, épaississement des parois des vésicules séminales...) [6].

\section{CONCLUSION}

La leucospermie n'est pas pathognomonique d'une pathologie définie. Elle ne peut être interprétée qu'en fonction du contexte, d'une part le contexte clinique à la recherche chez le patient, ainsi que chez sa partenaire, d'une symptomatologie uro-génitale présente ou ancienne et d'autre part le contexte biologique avec l'ensemble des analyses du sperme et des analyses bactériologiques de la partenaire.

Il est important de s'assurer d'une excellente communication entre clinicien qui prend en charge le patient et le laboratoire afin que la prise en charge médicale soit la mieux adaptée et la moins iatrogène possible.

Enfin il ne faut pas oublier le propriétaire du sperme à qui l'on doit expliquer simplement les résultats de ses analyses.

\section{REFERENCES}

1. AUGER J. : Dérivés actifs de l'oxygène et dysfonctions spermatiques : rôle de l'infection du tractus génital de l'homme? Andrologie, 1998, 8 : 234-244.

2. GIL T., CASTILLA J.A., HORTAS M.L, et al. : CD4+ cells in human ejaculates. Hum. Reprod., 1995, 10 : 2923-2327.

3. KIESSLING A.A., LAMPARELLI N., YIN HZ, et al. : Semen leukocytes : friends or foes ? Fertil. Steril., 1995, $64:$ 196-198.

4. LUCAS H. : Inflammation de l'appareil génital masculin et reproduction : traitement in vitro. Andrologie, 1998, $8: 269-279$.

5. SIGMAN M., LOPES L. : The correlation between round cells and white blood cells in the semen. J. Urol., 1993, 149 : 1338-1340.

6.STAERMAN F. : Inflammation de l'appareil génital masculin et reproduction : situation clinique. Andrologie, 1998, 8 : 254-258.

7. VENDRELY E. : Identification des cellules rondes du sperme. Andrologie, 1998, $8: 280-286$.

8. WHO laboratory manual for the examination of human semen and sperm-cervical mucus interaction, edition 1992.

9. WOLFF H ., ANDERSON D.J. : Immunohistologic characterization and quantitation of leucocytes subpopulaion in human semen. Fertil. Steril., 1988, 49 : 497504.

10. WOLFF H., ANDERSON D.J : Evaluation of granulocyte elastase as a seminal plasma marker for leukocytosperma. Fertil. Steril., 1988, $50: 129-132$.

11. WOLFF H. : Methods for the detection of male genital tract inflammation. Andrologia, 1998, 30 (Suppl.1) : 35-39.

\author{
ABSTRACT \\ Leukocytospermia in routine clinical \\ practice
}

A. CHOCAT

In man, leukocytes, usually neutrophil polymorphonuclear cells, are present in most ejaculates. The presence of large numbers of leukocytes is suggestive of inflammation of the genital tract, possibly secondary to infection. Routine quantification of these cells is not 
easy and is based on a combination of analysis of fresh semen, and after detection of intracellular peroxidases characteristic of polymorphonuclear cells, and then on stained smears.

The presence of a high proportion of altered leukocytes is highly suggestive of infection. Although a large number of leukocytes in the semen reflects the presence of inflammation, the role of these granulocytes, present in small numbers, is still controversial. Leukocytospermia must be interpreted in the light of the patient's clinical and laboratory findings, based on good communication between the various health care professionals involved.

Key words : Leukocytospermia, male infertility, infection, inflammations 